\title{
Cardiac Three-Dimensional Printing Using Noninvasive Modalities: Will It Revolutionize Cardiac Care?
}

\author{
Amier Ahmad ${ }^{1}$ Navin C. Nanda ${ }^{2}$ Thomas Bartel ${ }^{3} \quad$ Samuel McElwee $^{2}$ \\ ${ }^{1}$ Tinsley Harrison Internal Medicine Program, University of Alabama \\ at Birmingham, Birmingham, Alabama, United States \\ 2 Division of Cardiovascular Disease, University of Alabama at \\ Birmingham, Birmingham, Alabama, United States \\ ${ }^{3}$ Heart and Vascular Institute, Cleveland Clinic Abu Dhabi, \\ Abu Dhabi, United Arab Emirates \\ J Card Crit Care TSS 2017;1:6-9.

\begin{abstract}
Address for correspondence Navin C. Nanda, MD, S102, Spain Wallace Building, 619 19TH Street South, AL 35294, United States (e-mail: nanda@uab.edu).
\end{abstract}

Abstract
Keywords
- three dimensional
printing
- computed
tomography
- noninvasive
modalities
- simulation
techniques
- cardiac care

Three-dimensional (3D) printing has emerged as a tool for clinicians to understand the underlying mechanisms and pathophysiology of these disorders in a simulated setting. Three-dimensional printing consists of manipulating a two-dimensional (2D) image obtained via noninvasive modalities, such as computed tomography (CT), magnetic resonance imaging (MRI), or 3D echocardiography, to a 3D dataset and then finally into a physical model. Three-dimensional printing allows for creation of specific models in a variety of diseases such as cardiac shunts that can be visualized by surgeons prior to device placement in a matter of hours. Further, cardiac tumors, which typically invade the myocardium, have been replicated to allow surgeons the ability to plan procedures prior to any incision. This creates the potential for propagation of error within the health field. Three-dimensional printing has emerged as a tool for clinicians to understand the underlying mechanisms and pathophysiology of these disorders in a simulated setting though standardization of the technique is yet awaited.

\section{Introduction}

Recent advancements in technology have brought threedimensional (3D) printing to the forefront of medicine. Three-dimensional printing consists of manipulating a two-dimensional (2D) image obtained via noninvasive modalities, such as computed tomography (CT), magnetic resonance imaging (MRI), or 3D echocardiography to a 3D dataset and then finally into a physical model. These models can then be used to enhance patient care in both acute and chronic clinical settings. Critical care medicine represents one area that has been influenced by the development of 3D printing. Multiple congenital abnormalities such as ventricular septal defect (VSD) or ventricular septal rupture following a myocardial infarction can be simulated prior to being witnessed in patients. Here, we summarize how 3D printing is currently being implemented in critical care medicine.

\section{Outlining Three-Dimensional Printing}

Three-dimensional printing (also known as rapid prototyping) is characterized by creating a 3D virtual object using a 3D image dataset in a process known as segmentation (-Fig. $\mathbf{1}$ ). These files are then transformed to physical models with the use of specialized printers. Multiple medical and surgical specialties have capitalized on this development to create patient-specific model. ${ }^{1}$ Traditionally, CT imaging was the only available modality transformable to 3D models. However, other forms of imaging have now emerged as potential sources for segmentation. Cardiac MRI has been used to
DOI https://doi.org/ $10.1055 / \mathrm{s}-0037-1604335$
Copyright (๑) 2017 Official Publication of License terms The Simulation Society (TSS), accredited by International Society of Cardiovascular Ultrasound (ISCU) 


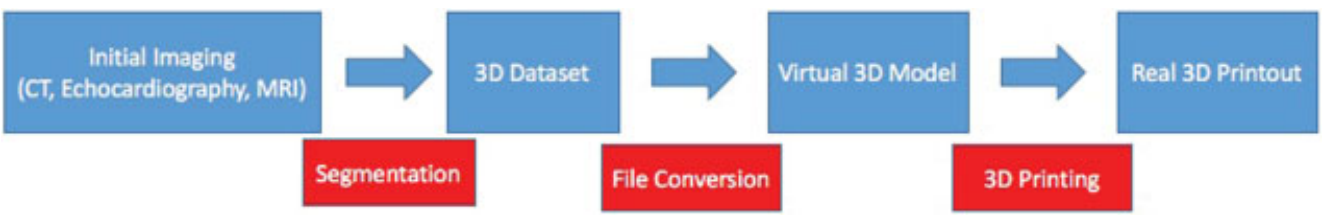

Fig. 1 Three-dimensional (3D) printing workflow. CT, computed tomography; MRI, magnetic resonance imaging (Reproduced with permission from Dr. Thomas Bartel).

simulate multiple congenital heart disorders, whereas 3D transesophageal echocardiography has been instrumental in replicating mitral valve pathology. 2,3

\section{Procedural Applications}

A variety of pathologies are encountered in a critical care setting. Historically, it has been difficult to train physicians for these clinical situations, outside of experiencing them firsthand. Three-dimensional printing has emerged as a tool for clinicians to understand the underlying mechanisms and pathophysiology of these disorders in a simulated setting (-Fig. 2). For instance, ventricular assist devices (VADs) remain an underutilized option in patients with heart failure, predominantly due to variable and complex anatomy that prohibits placement of the device. ${ }^{4}$ Three-dimensional printing allows for creation of specific models that can be visualized by surgeons prior to device placement in a matter of hours. ${ }^{5}$ Furthermore, cardiac tumors, which typically invade the myocardium, have been replicated to allow surgeons the ability to plan procedures prior to any incisions. ${ }^{6}$ Three-dimensional models can clearly differentiate boundaries between these intracardiac tumors and the myocardium. ${ }^{7}$ Complex congenital diseases such as atrial septal defect, VSD, or tetralogy of Fallot can be visualized prior to repair ( - Fig. 3)..$^{8-13}$

Catheter-based procedures have also benefited from 3D printing. Models of complex coronary vasculature for patients admitted following myocardial infarction can be created prior to interventional procedures. ${ }^{14}$ Perfusion hemodynamics and

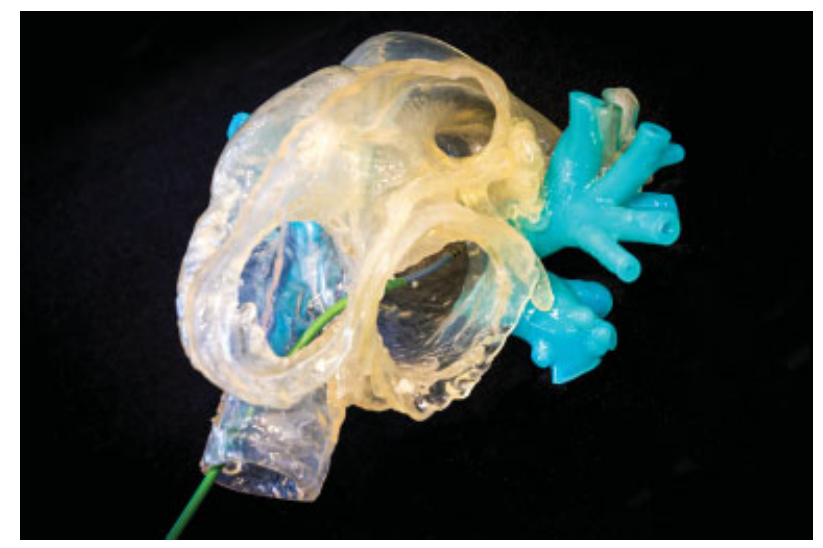

Fig. 2 Simulation model demonstrating catheter ablation for atrial fibrillation. The catheter is visualized entering the left atrium after transseptal puncture. Pulmonary veins are colored blue. stenosis of vessels can be visualized in full before and after an intervention is made. ${ }^{15}$ Valvular pathologies including aortic stenosis and mitral regurgitation can be modeled for preprocedural planning. Transcatheter aortic valve repair for aortic regurgitation is complicated by the spectrum of flow dynamics that exist with this condition. Three-dimensional printing allows for creation of specific models that highlight patientspecific flow hemodynamics (- Fig. 4). ${ }^{15}$ Three-dimensional modeling for percutaneous mitral valve repair allows clinicians to gauge the proper valve size needed for repair, in addition to whether further imaging would be needed. ${ }^{16}$ Another potential use of 3D printing is in the placement of the extracorporeal membrane oxygenation device by cardiac

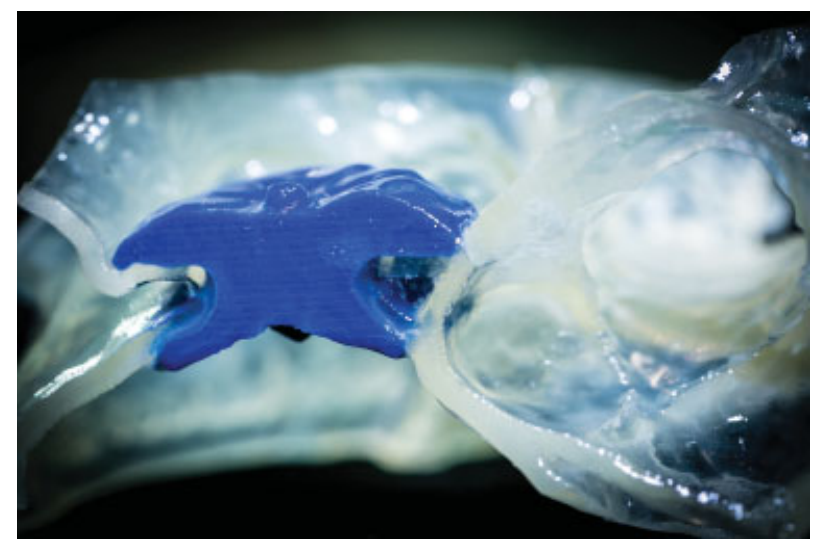

Fig. 3 Atrial septal defect closure. The closure device, viewed from the side, is colored blue.

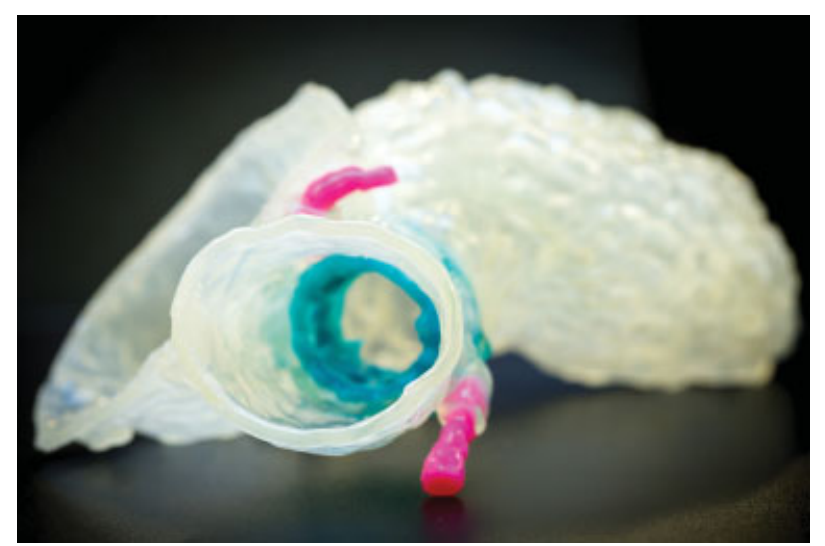

Fig. 4 Model of a transcatheter aortic valve replacement. The artificial valve is colored blue. The coronary vessels are colored pink. 

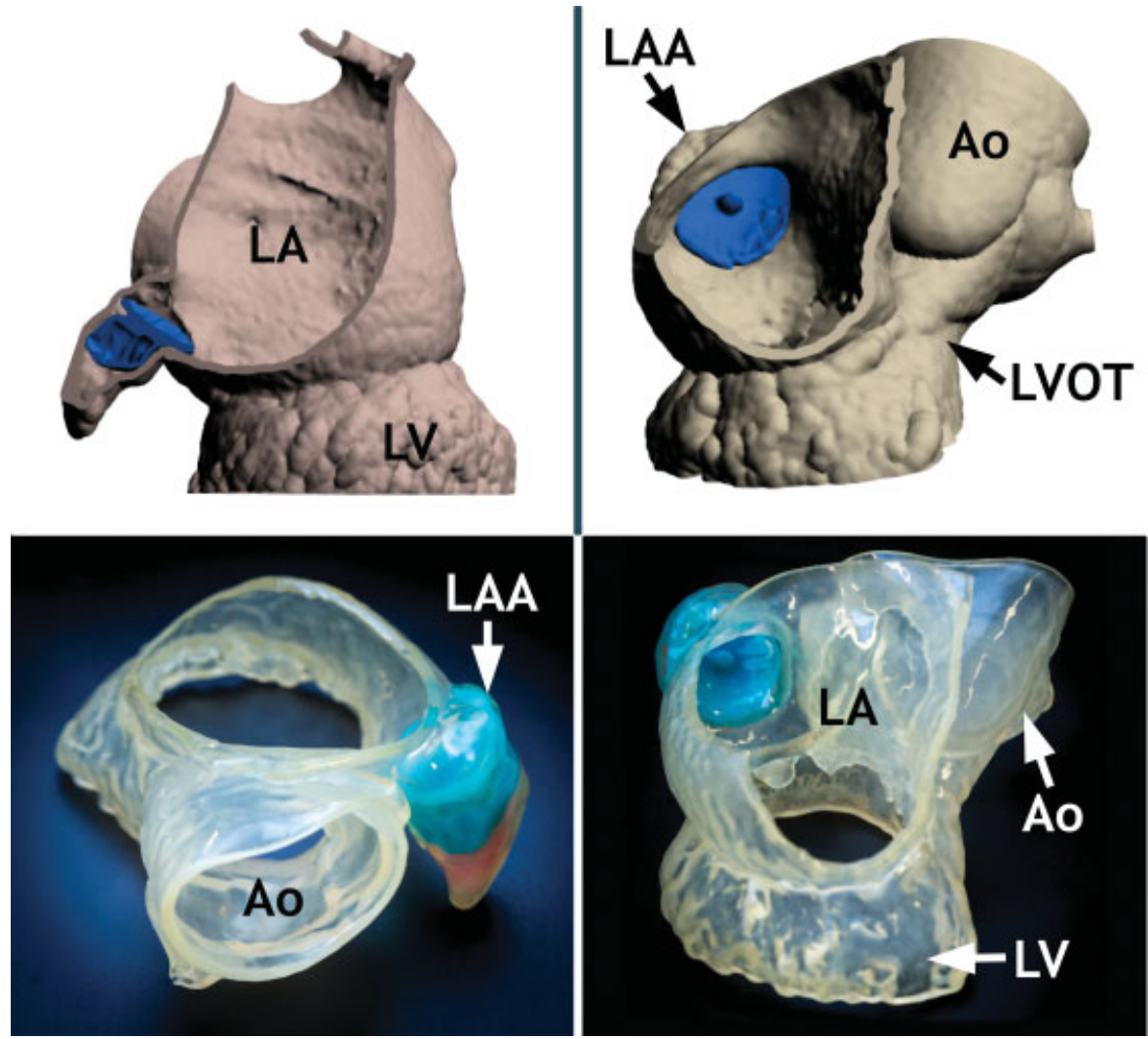

Fig. 5 Left atrial appendage closure. The upper panels depict the virtual 3D modeling showing closure. Bottom panels depict the actual 3D printout. The closure device is colored blue. Ao, aorta; LA, left atrium; LAA, left atrial appendage; LV, left ventricle; LVOT, left ventricular outflow tract.

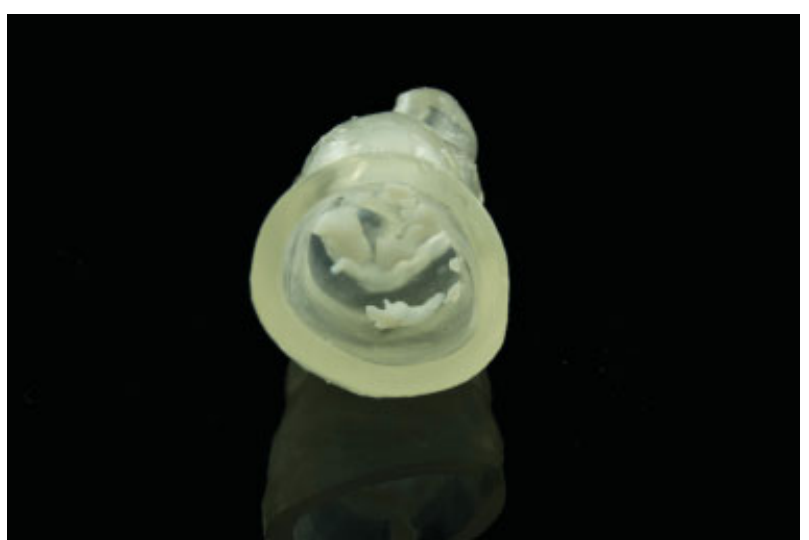

Fig. 6 Model of a calcified aortic valve.

anesthesiologists in intensive care units, as well as assisting in left atrial appendage closures to minimize stroke risk in patients with atrial fibrillation (- Fig. 5).

Outside of specific interventions, 3D modeling allows for training of medical professionals. Complex structural anatomy is easily seen and specific pathologic changes can be demonstrated ( - Fig. $\mathbf{6}$ ). In addition, 3D models can be used to educate patients on complicated visual-spatial anatomy prior to procedures. Data suggest that the use of these models enhances the patient-provider relationship. ${ }^{8,12}$

\section{Conclusion}

Three-dimensional printing has become increasingly more popular in the field of medicine. It allows for the clinicians to develop comprehensive models of complicated anatomy and physiology. These 3D models are then used for preprocedural planning, understanding and visualizing complex pathology, and facilitate discussion with other health professionals and patients. Also, they serve as a source of quality control because potential surgical results can be immediately visualized.

Although 3D printing represents a promising technological advancement in medicine, certain issues remain that need to be addressed. Currently, the supplies and techniques involved in 3D printing are not standardized. Multiple types of materials can be used to create models, in addition to the plethora of printer types and imaging modalities that can be used to create 3D image sets. This creates the potential for propagation of error within the health field. Furthermore, cost associated with creating these models varies widely depending on the complexities of the anatomy and physiology being replicated. Literature regarding the utility of 3D modeling has also only been based on small case reports. Whether 3D modeling and imaging results in an improvement in patient outcomes remains to be seen. ${ }^{17}$

Overall, 3D printing represents a promising new area of advancement in the field of medicine and critical care. Further development and refinement of 3D printing together 
with reduction in its cost will certainly contribute to enhancement of patient care and could change the way cardiology and cardiac surgery are practiced today.

\section{Acknowledgment}

All figures in this manuscript are reproduced with permission of Dr. Thomas Bartel.

\section{References}

1 Byrne N, Velasco Forte M, Tandon A, Valverde I, Hussain T. A systematic review of image segmentation methodology, used in the additive manufacture of patient-specific 3D printed models of the cardiovascular system. JRSM Cardiovasc Dis 2016;5:2048004016645467

2 Schievano S, Migliavacca F, Coats L, et al. Percutaneous pulmonary valve implantation based on rapid prototyping of right ventricular outflow tract and pulmonary trunk from MR data. Radiology 2007;242(02):490-497

3 Sodian R, Weber S, Markert M, et al. Stereolithographic models for surgical planning in congenital heart surgery. Ann Thorac Surg 2007;83(05):1854-1857

4 Gelow JM, Song HK, Weiss JB, Mudd JO, Broberg CS. Organ allocation in adults with congenital heart disease listed for heart transplant: impact of ventricular assist devices. J Heart Lung Transplant 2013;32(11):1059-1064

5 Farooqi KM, Nielsen JC, Uppu SC, et al. Use of 3-dimensional printing to demonstrate complex intracardiac relationships in double-outlet right ventricle for surgical planning. Circ Cardiovasc Imaging 2015;8(05):8

6 Jacobs S, Grunert R, Mohr FW, Falk V. 3D-Imaging of cardiac structures using 3D heart models for planning in heart surgery: a preliminary study. Interact Cardiovasc Thorac Surg 2008;7(01):6-9

7 Schmauss D, Haeberle S, Hagl C, Sodian R. Three-dimensional printing in cardiac surgery and interventional cardiology: a single-centre experience. Eur J Cardiothorac Surg 2015;47(06): 1044-1052
8 Garekar S, Bharati A, Chokhandre M, et al. Clinical application and multidisciplinary assessment of three dimensional printing in double outlet right ventricle with remote ventricular septal defect. World J Pediatr Congenit Heart Surg 2016;7(03): 344-350

9 Olivieri LJ, Krieger A, Loke YH, Nath DS, Kim PC, Sable CA. Threedimensional printing of intracardiac defects from three-dimensional echocardiographic images: feasibility and relative accuracy. J Am Soc Echocardiogr 2015;28(04):392-397

10 Costello JP, Olivieri LJ, Su L, et al. Incorporating three-dimensional printing into a simulation-based congenital heart disease and critical care training curriculum for resident physicians. Congenit Heart Dis 2015;10(02):185-190

11 Ryan JR, Moe TG, Richardson R, Frakes DH, Nigro JJ, Pophal S. A novel approach to neonatal management of tetralogy of Fallot, with pulmonary atresia, and multiple aortopulmonary collaterals. JACC Cardiovasc Imaging 2015;8(01):103-104

12 Kiraly L, Tofeig M, Jha NK, Talo H. Three-dimensional printed prototypes refine the anatomy of post-modified Norwood-1 complex aortic arch obstruction and allow presurgical simulation of the repair. Interact Cardiovasc Thorac Surg 2016;22(02): 238-240

13 Bartel T, Rivard A, Jimenez A, Edris A. Three-dimensional printing for quality management in device closure of interatrial communications. Eur Heart J Cardiovasc Imaging 2016;17(09):1069

14 Javan R, Herrin D, Tangestanipoor A. Understanding spatially complex segmental and branch anatomy using 3d printing: Liver, lung, prostate, coronary arteries, and circle of Willis. Acad Radiol 2016;23(09):1183-1189

15 Vukicevic M, Mosadegh B, Min JK, Little SH. Cardiac 3d printing and its future directions. JACC Cardiovasc Imaging 2017;10(02): $171-184$

16 Altiok E, Becker M, Hamada S, et al. Real-time 3D TEE allows optimized guidance of percutaneous edge-to-edge repair of the mitral valve. JACC Cardiovasc Imaging 2010;3(11):1196-1198

17 Bartel T, Rivard A, Jimenez A, Mestres CA, Müller S. Medical threedimensional printing opens up new opportunities in cardiology and cardiac surgery. Eur Heart J 2017. Doi: 10.1093/eurheartj/ ehx016 